\begin{tabular}{|c|c|}
\hline Title & A simple drain current model for single walled carbon nanotube network thin-film transistors \\
\hline Author(s) & Sano, Eiichi; Tanaka, Tomo \\
\hline Citation & $\begin{array}{l}\text { Journal of A pplied Physics, 115(15), } 154507 \\
\text { https://doi.org/10.1063/1.4871775 }\end{array}$ \\
\hline Issue Date & $2014-04-17$ \\
\hline Doc URL & http:/hdl.handle.net/2115/56755 \\
\hline Rights & 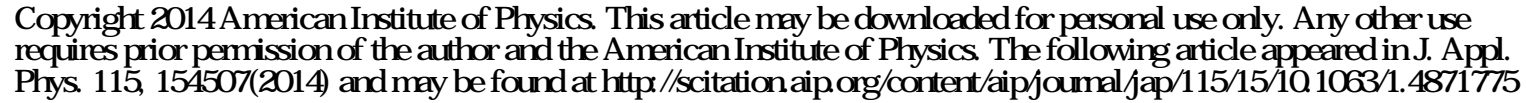 \\
\hline Type & article \\
\hline File Information & 1.4871775.pdf \\
\hline
\end{tabular}

Instructions for use 


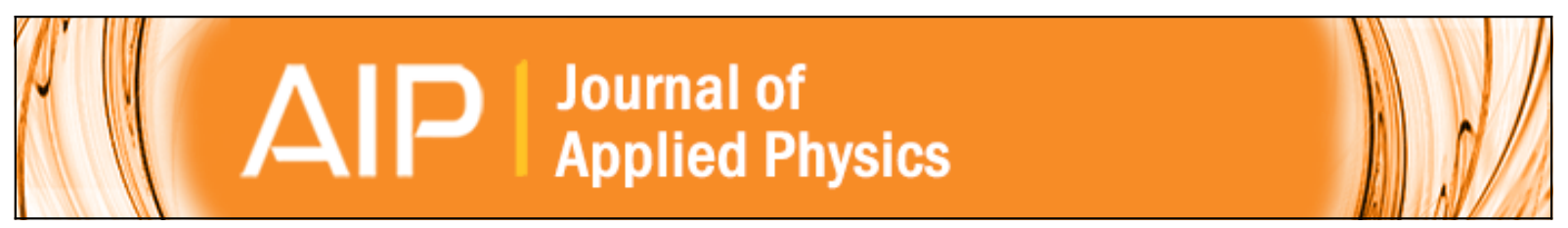

\section{A simple drain current model for single-walled carbon nanotube network thin-film transistors}

Eiichi Sano and Tomo Tanaka

Citation: Journal of Applied Physics 115, 154507 (2014); doi: 10.1063/1.4871775

View online: http://dx.doi.org/10.1063/1.4871775

View Table of Contents: http://scitation.aip.org/content/aip/journal/jap/115/15?ver=pdfcov

Published by the AIP Publishing

\section{Articles you may be interested in}

Static and low frequency noise characterization of N-type random network of carbon nanotubes thin film transistors

J. Appl. Phys. 114, 154503 (2013); 10.1063/1.4825221

Solution processable carbon nanotube network thin-film transistors operated in electrolytic solutions at various $\mathrm{pH}$

Appl. Phys. Lett. 101, 223101 (2012); 10.1063/1.4768683

Low-frequency noise in amorphous indium-gallium-zinc oxide thin-film transistors from subthreshold to saturation Appl. Phys. Lett. 97, 122104 (2010); 10.1063/1.3491553

A Comprehensive Model for Low Frequency Noise in PolySi ThinFilm Transistors

AIP Conf. Proc. 772, 1489 (2005); 10.1063/1.1994678

$1 / f$ noise modeling in long channel amorphous silicon thin film transistors

J. Appl. Phys. 87, 1983 (2000); 10.1063/1.372124

\section{APP $\left.\right|_{\text {Applied Physics }} ^{\text {Journal of }}$}

Journal of Applied Physics is pleased to announce André Anders as its new Editor-in-Chief 


\title{
A simple drain current model for single-walled carbon nanotube network thin-film transistors
}

\author{
Eiichi Sano ${ }^{\text {a) }}$ and Tomo Tanaka \\ Research Center for Integrated Quantum Electronics, Hokkaido University, Sapporo 060-8628, Japan
}

(Received 9 December 2013; accepted 8 April 2014; published online 17 April 2014)

\begin{abstract}
Single-walled carbon nanotube (CNT) network thin-film transistors (TFTs) are attractive owing to their simple, low-cost fabrication methods. However, the detailed operation mechanism for TFTs is still unclear. In this paper, we present a simple model for the drain current of CNT network TFTs operated in the linear region. The model is based on the gate electrostatics and the continuity condition of the currents through CNT and CNT-CNT tunnel junction. The model is evaluated by comparing its calculations to experimentally measured drain current and low frequency $(1 / f)$ noise parameters. Even though the present model is based on simplified assumptions, it provides useful information to improve the TFT performance. (C) 2014 AIP Publishing LLC.
\end{abstract}

[http://dx.doi.org/10.1063/1.4871775]

\section{INTRODUCTION}

Carbon nanotubes (CNTs) have been attracting much attention due to wide application areas thanks to their excellent electric, mechanical, and thermal properties. ${ }^{1}$ Among the properties, the unique one-dimensional conduction and extremely high carrier mobility in single-walled (SW) CNTs make them a candidate material for future field-effect transistors (FETs) beyond silicon complementary metaloxide-semiconductor (CMOS) technology., ${ }^{2,3}$ However, the precise control of fabricating CNTs with an appropriate chirality and placing individual CNTs on a substrate remains unsolved. CNT networks have been explored as an alternative solution for transistor channels. ${ }^{4-12}$ Although CNT network thin-film transistors (TFTs) are outperformed by individual CNT FETs, they provide a simple and low-cost fabrication method with "writing" transistors by an inkjet printer. ${ }^{7}$ Small-scaled integrated circuits with p-channel configuration ${ }^{11}$ as well as an complementary inverter ${ }^{5}$ have been successfully fabricated.

Usual CNT networks are random networks composed of metallic and semiconducting CNTs. The performance of CNT TFTs strongly depends on the network topology. For example, a conduction path composed of metallic CNTs increases the leakage current and degrades the current ON/OFF ratio. The relationship between network topology and TFT performance such as percolation conduction and ON/OFF ratio has been extensively investigated both experimentally and theoretically. ${ }^{6,8-10}$ However, the resistance values measured for CNT-CNT junctions ${ }^{13}$ were used in these Monte Carlo simulations. Despite the network complexity, the drain current-voltage curves for CNT TFTs are very similar to those for silicon MOSFETs. The "effective mobility"

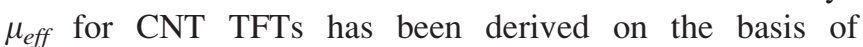
MOSFET theory, $g_{m}=\frac{W}{L} \mu_{e f f} C_{O X}\left(V_{G S}-V_{t h}\right) V_{D S}$ ( $g_{m}$ : transconductance, $W$ : gate width, $L$ : gate length, $C_{O X}$ : gate capacitance per unit area, $V_{G S}$ : gate voltage, $V_{D S}$ : drain voltage,

${ }^{a)}$ Electronic mail: esano@rciqe.hokudai.ac.jp and $V_{t h}$ : threshold voltage). The reasons for the similarity and the factor determining the effective mobility are still unclear. The detailed operation mechanism for CNT TFTs must be clarified to improve their performances.

In this paper, we present a simple drain current model for CNT network TFTs. The model is based on the gate electrostatics and the continuity condition of the currents through CNT and CNT-CNT tunnel junction. The linear region in the drain current is focused on, but the saturation region is out of the scope of this paper. The formulae are presented for $\mathrm{n}$-channel TFTs. The model is evaluated by comparing the calculations with experimentally measured drain current and low frequency $(1 / f)$ noise parameter. The temperature assumed here is $300 \mathrm{~K}$.

\section{MODEL}

\section{A. CNT}

Since CNTs may be either semiconducting (S) or metallic (M) depending on their chirality, the feasible combinations of CNT-CNT junctions in a conduction path are S-S, $\mathrm{M}-\mathrm{M}$, and M-S. Among them, M-S junction has much larger resistance than M-M and S-S junctions, ${ }^{13}$ and the contribution of M-S junction on the current conduction is negligibly small compared with those of other junctions. To reduce the leakage current, formation of a metallic path should be avoided. This may be achieved by carefully using semiconducting CNT solution with purity of $98 \%$ or $99 \%$. Therefore, only S-S junction is taken into account in our model. Although CNTs with different chirality are included in a CNT TFT, we assumed that all the CNTs are equivalent and are the zigzag type with chiral index of $(m, 0)$ to simplify the model calculation. Note that the integer $m$ not $n$ in usual chiral index of $(n, m)$ is used to avoid misunderstanding because $n$ will be used for the electron density below. The density of states is given by ${ }^{2,14}$

$$
D(E)=\sum_{i} \frac{8}{3 \pi a_{C-C} t} \frac{E}{\sqrt{E^{2}-E_{i}^{2}}} \theta\left(E-E_{i}\right),
$$


with

$$
E_{i}=\frac{3 a_{C-C} t}{d}\left|i-\frac{2 m}{3}\right|
$$

where $a_{C-C}$ is the C-C bond length $(0.144 \mathrm{~nm}), t$ is the transfer integral $(\sim 3 \mathrm{eV}), d$ is the diameter of CNT $\left(=\sqrt{3} m a_{C-C} / \pi\right)$, and $\theta$ is the step function. The electron density is given by

$$
n\left(E_{f}\right)=\int_{0}^{\infty} \frac{D(E)}{2} f\left(E-E_{f}\right) d E .
$$

Here, the energy $E$ and Fermi energy $E_{f}$ are measured from the middle of the bandgap. Figure 1 shows the calculated electron density as a function of $E_{f}$ where $m=20$ and $d=1.57 \mathrm{~nm}$.

\section{B. Modulation of potential difference between crossed two CNTs}

The potential difference between crossed two CNTs can change with the gate voltage in a TFT. The modulation of the potential difference with the gate voltage was investigated by numerically solving a three-dimensional Poisson equation. To simplify the calculation, the CNT was treated as a solid square column instead of a real hollow cylinder, and the electron density in the Poisson equation was calculated with (3). Since the electron density depends on the potential, the Poisson equation was self-consistently solved. The Fermi energy $E_{f}$ was assumed to be 0 . Figure 2(a) shows an example of the cross-sectional view of potential distribution calculated for orthogonally crossed CNTs (indicated by the dotted lines). The gate $\mathrm{SiO}_{2}$ thickness $t_{O X}$ (distance from the gate electrode to the upper CNT) was $10 \mathrm{~nm}$, while the distance between the lower CNT and the ground plane was $200 \mathrm{~nm}$. The distance between the two CNTs was $0.4 \mathrm{~nm}$. The change in the potential difference between the two CNTs with the gate voltage is shown in Fig. 2(b). In this case, the potential difference was approximated by $V_{\text {gap }}=0.022 V_{G S}$. We assumed that the potential difference between the two CNTs depended on the average gate electric field $\left(V_{G S} / t_{O X}\right)$ and was given by

$$
V_{\text {gap }}=0.22 \frac{V_{G S}}{t_{O X}},
$$

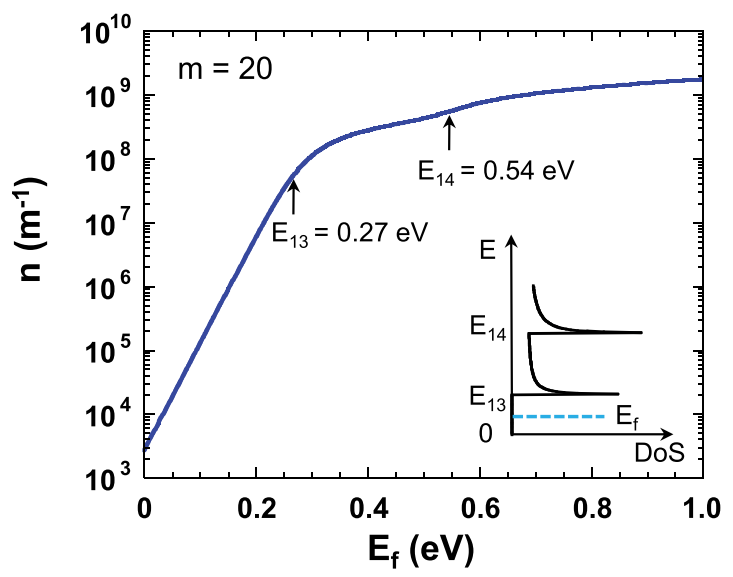

FIG. 1. Electron density as a function of $E_{f}$. Insets: Density-of-states (DoS).

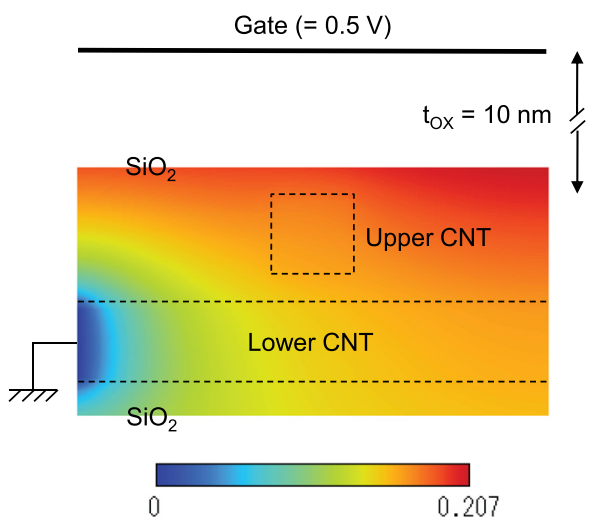

(a)

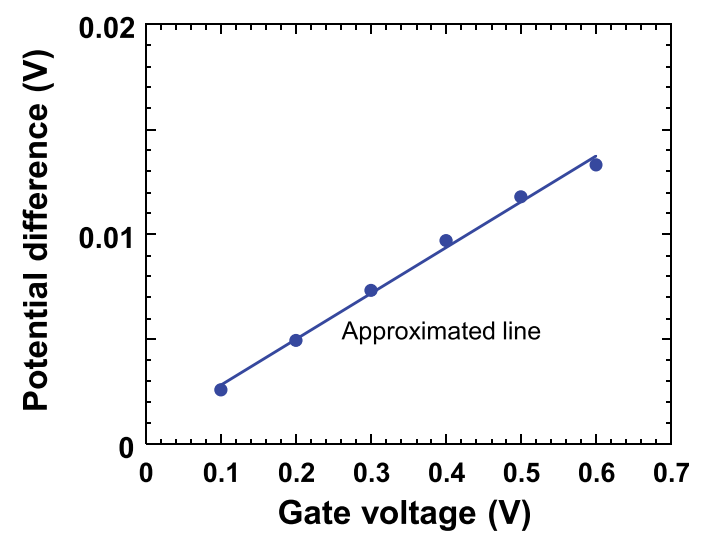

(b)

FIG. 2. (a) Cross-sectional view of potential distribution calculated for orthogonally crossed CNTs and (b) calculated potential difference as a function of the gate voltage. One end of the bottom CNT was grounded. The dotted lines indicate the outlines of model CNTs.

where $t_{O X}$ is in the unit of $\mathrm{nm}$. The potential drop in the lower CNT might have been caused by the boundary conditions in which its left end was grounded, the other end was open, and both ends of the upper CNT were open. In an actual TFT, the Fermi energy difference between the two CNTs in a current path may be small and the potential difference may be smaller than these calculations. Despite this presumption, the above relationship (4) was used not to rule out the effect of the potential difference modulation by the gate voltage on the drain current.

\section{Tunnel current between two CNTs}

The CNT-CNT junctions in the CNT network may be the bottleneck of the current path from drain to source in a TFT. The tunnel current between two CNTs is given by

$$
I_{T}=\frac{2 e}{h} \int_{0}^{\infty} T(E)\left[f_{L}\left(E-E_{f L}\right)-f_{R}\left(E-E_{f R}\right)\right] d E,
$$

where $e$ is the elementary charge, $h$ is the Planck's constant, $f_{L(R)}$ is the Fermi function, the subscripts $L$ and $R$ mean the left and right CNTs, and $T(E)$ is the transmission coefficient through the barrier between the two CNTs. The transmission coefficient is approximated as 


$$
T(E)=\exp \left[-2 L_{B} \sqrt{2 m^{*}\left(\phi_{B}-E\right)} / \hbar\right],
$$

where $L_{B}$ is the barrier width (distance between the two CNTs), $m^{*}$ is the effective mass of electron, and $\phi_{B}$ is the barrier height. Although the WKB (Wrentzel-Kramers-Brillouin) method used here would not be only for the CNT-CNT junction, our interest was to evaluate the method to see if it reproduces measured tunnel currents and junction resistances. Figure 3 compares measured ${ }^{13}$ and calculated tunnel current. In the calculation, $\phi_{B}=4.8 \mathrm{eV}$ (work function of $\mathrm{SWCNT}^{15}$ ), $m^{*}=0.25 m_{0}$ ( $m_{0}$ : mass of free electron), $E_{f}=0.35 \mathrm{eV}$ (measured from the midgap), and $L_{B}=0.31 \mathrm{~nm} .{ }^{16}$ Note that the Fermi energy of $0.35 \mathrm{eV}$ corresponds to the hole conduction in CNTs when Au or Pd is used for electrodes. The calculated tunnel current agreed with that measured by Fuhrer et al..$^{13}$ Nirmalraj et al ${ }^{17}$ and Znidarsic et al. ${ }^{18}$ measured the junction resistances of $98 \mathrm{k} \Omega-2.7 \mathrm{M} \Omega$ and $29-532 \mathrm{k} \Omega$, respectively, for SWCNTs and SWCNT bundles. The former reported that the junction resistance increased as the diameters of SWCNTs or bundles increased, while the latter reported the opposite dependence. Figure 4 shows the junction resistance calculated by (5) and (6) with $E_{f}$ of $0.35 \mathrm{eV}$ for zigzag-type semiconducting CNTs (from CNT1 to CNT2). When CNT1 has a larger diameter than CNT2 $\left(d_{1}>d_{2}\right)$, the electrons with energy lower than the conduction-band edge of CNT2 are prohibited from tunnelling and the junction resistance is high. When $d_{1}$ becomes smaller, the energy difference between the Fermi energy and the conduction-band edge becomes larger and so does the junction resistance. The calculations showed that the junction resistance decreased as the diameters of CNTs increased. The calculated resistance ranged from $370 \mathrm{k} \Omega$ to $1.98 \mathrm{M} \Omega$ for the SWCNTs with $d$ of $1.1-2.0 \mathrm{~nm}$. Although the chirality might differ from those of SWCNTs used in the experiments, the present model well reproduced the measured junction resistances. The calculations predicted that the junctions between two CNTs with similar energy bandgaps are dominant even if CNTs with a variety of chirality are included in CNT networks.

Figure 5 shows a schematic diagram of the cross section along a current path. When a positive gate-source voltage $V_{G S}$ and drain-source voltage $V_{D S}$ are applied, the junctions between CNTs $a$ and $b$ and CNTs $b$ and $c$ have different

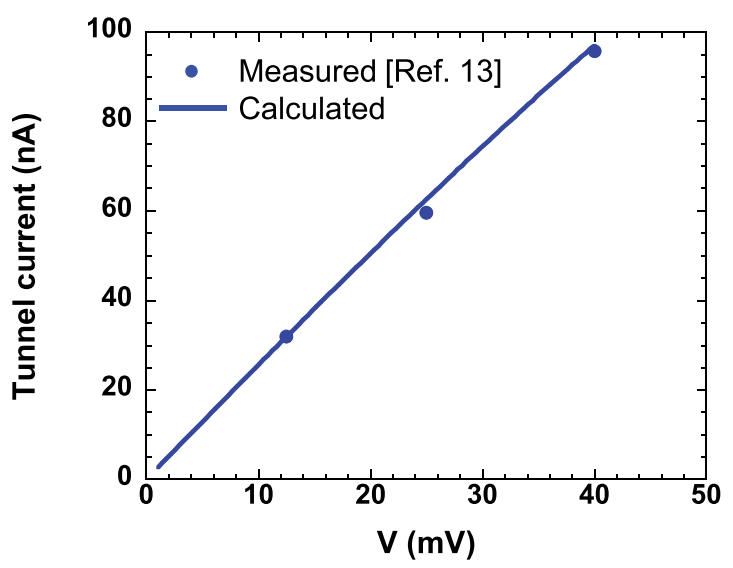

FIG. 3. Comparison between measured ${ }^{13}$ and calculated tunnel current.

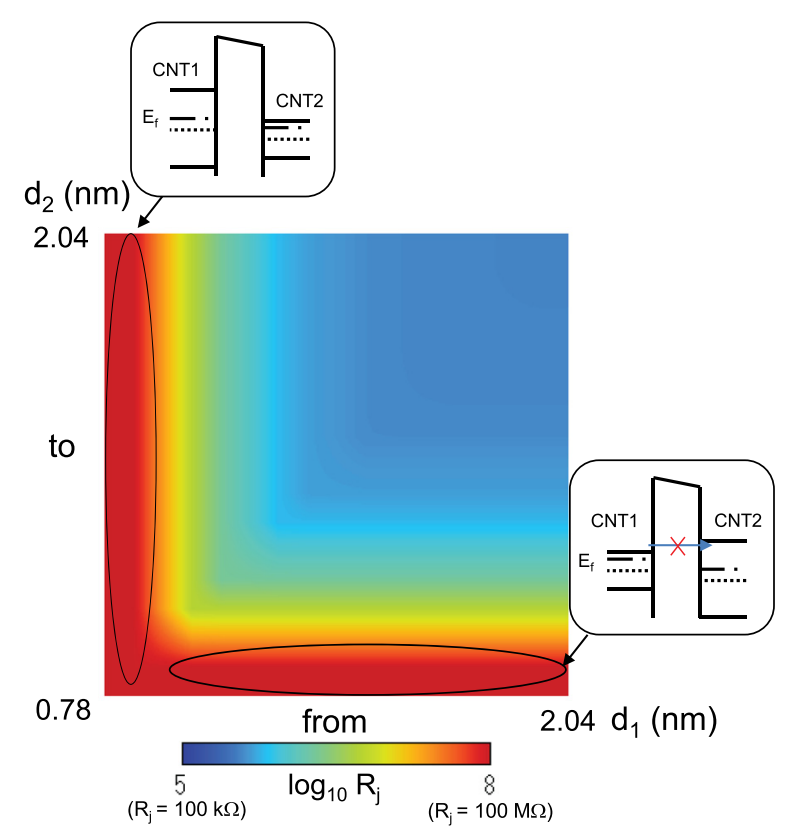

FIG. 4. Junction resistance between two CNTs with different diameters.

potential distributions. The upper CNTs $a$ and $c$ have lower electron energy than the lower CNT $b$, while the Fermi energy $E_{f}$ gradually decreases toward the drain. In the case of the junction between CNTs $b$ and $c$, the tunnel current is independent of the energy difference between CNTs $b$ and $c$. On the other hand, the tunnel current between CNTs $a$ and $b$ depends on the energy difference because the electron with energy lower than the lowest energy level [given by (2)] of CNT $b$ cannot tunnel from CNTs $a$ to $b$. Figure 6 shows the calculated tunnel current versus Fermi energy characteristics as a function of the energy difference between two CNTs $E_{\text {gap }}$ (left-right). As the absolute value of $E_{\text {gap }}$ increases, the amount of the electrons inhibited from tunneling increases and the tunnel current decreases. The calculated results suggested that a current ON/OFF ratio of more than $10^{4}$, which has been observed in CNT TFTs, cannot be achieved only by changing the energy difference between two CNTs, $E_{\text {gap }}$, with the gate voltage and that the variation in the difference between the "surface potential" and the Femi energy with changing the gate voltage has a strong influence.

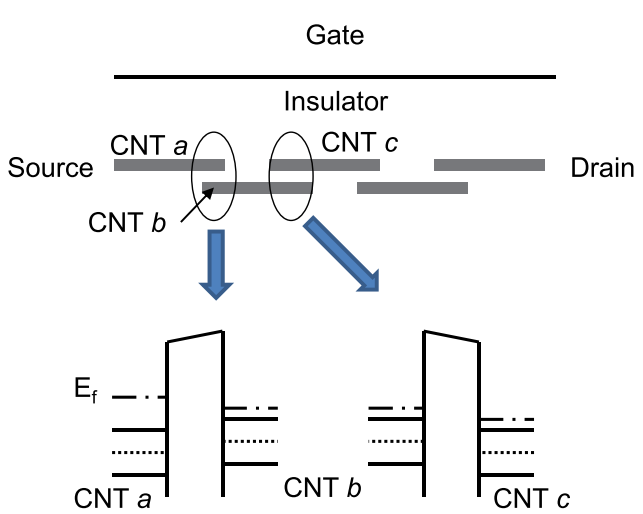

FIG. 5. Schematic diagram of cross section along a current path and electron energy distributions around the junctions $a-b$ and $b-c$. 


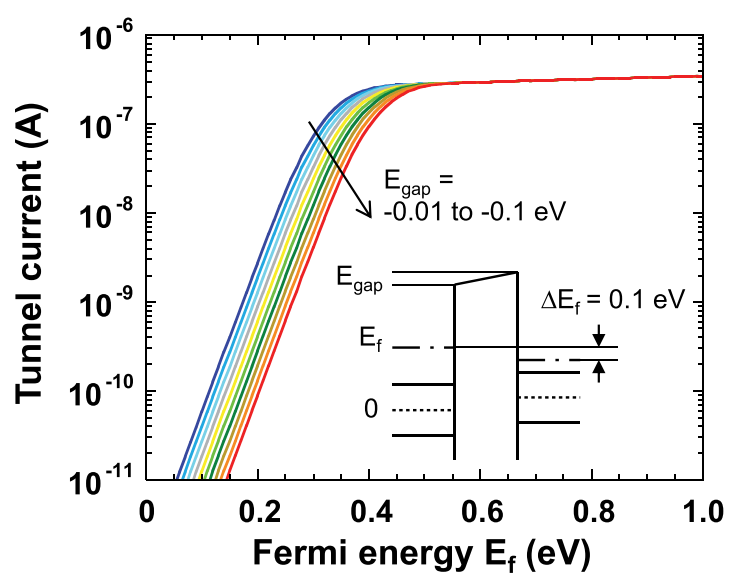

FIG. 6. Calculated tunnel current versus Fermi energy characteristics as a function of the energy difference between two CNTs $E_{\text {gap }}$. The Fermi energy difference $\Delta E_{f}$ is kept constant.

\section{Gate electrostatics}

The gradual channel approximation and the charge neutrality condition were used in our model. The charge neutrality condition for a CNT in a current path leads to

$$
C_{G S}\left(V_{G S}-V_{F B}-\phi_{S}\right)=e\left[n\left(E_{f}\right)-p\left(E_{f}\right)\right]
$$

where $V_{F B}$ is the flat-band voltage, $\phi_{S}$ is the surface potential (=the midgap energy of CNT), $n\left(E_{f}\right)$ is the electron density given by (3), and $p\left(E_{f}\right)$ is the hole density similarly calculated by (3). $C_{G S}$ is the gate capacitance given by

$$
C_{G S}=\left(C_{q}^{-1}+C_{O X}^{-1}\right)^{-1},
$$

where $C_{q}$ is the quantum capacitance of CNT and $C_{O X}$ is the gate oxide capacitance. The gate oxide capacitance for an individual CNT is approximated by

$$
C_{O X i}=\frac{2 \pi \varepsilon_{O X} \varepsilon_{0}}{\ln \left(t_{O X} / d\right)},
$$

where $\varepsilon_{O X}$ is the relative permittivity of the oxide, $\varepsilon_{0}$ is the permittivity of vacuum, $t_{O X}$ is the oxide thickness, and $d$ is the diameter of the CNT. Since individual CNTs and/or CNT bundles are located randomly in a CNT network, the actual value of the gate oxide capacitance may differ greatly from the value calculated by (9). The gate oxide capacitance for a CNT bundle was estimated with a simple model. Figure 7 shows a cross-sectional view of the CNT bundle model. All the CNTs are assumed to have an identical diameter and to be closely coupled to form a bundle with $l \mathrm{CNTs}$ on the line passing through the center of the bundle circle. The gate oxide capacitance for the bundle, $C_{\text {bundle, was calculated by (9) }}$ by replacing $d$ with the diameter of the bundle. When two bundles $A$ and $B$, schematically shown in Fig. 7, form a junction in a current path, however, the tunnel current between only two nearest CNTs (indicated by solid circles) may be dominant. Since the amount of the electric flux lines terminating at the half circle of the dominant CNT in the bundle is approximately $1 /(2 l)$ times the total amount of the electric flux lines terminating at the circumference of the bundle, the

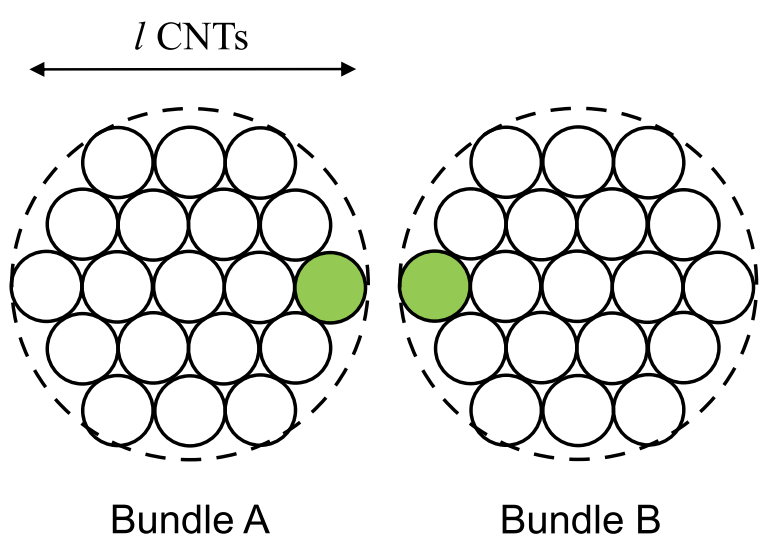

FIG. 7. Cross-sectional view of the CNT bundle model.

gate oxide capacitance for the dominant CNT is approximated by $C_{\text {bundle }} /(2 l)$. Dispersing individual CNTs in a solution is quite difficult, and CNTs tend to form bundles with sizes of $10-25 \mathrm{~nm}$ even if a surfactant is used. ${ }^{19-21}$ These sizes correspond to $l$ of more than 10 .

Another factor to be considered is a possible situation in which multiple CNTs and/or CNT bundles coexist in the network but not all of them contribute to the current conduction. The capacitance for the CNT contributing to current conduction is difficult to calculate exactly due to the randomness of the CNT network. Here, we estimated the effect of multiple CNT bundles by using the capacitance of a CNT array. The capacitance of a CNT array with a density of $D$ is given by ${ }^{22}$

$$
C_{\text {array }}=\frac{2 \pi \varepsilon_{O X} \varepsilon_{0} D}{\ln \left(\frac{\sinh \left(2 \pi t_{O X} D\right)}{\pi d D / 2}\right)}
$$

When $D^{\prime}$ CNTs contribute to the current conduction, a factor of $D^{\prime} / D$ is multiplied to (10) for calculating the gate capacitance. In addition, $\varepsilon_{O X}$ in (10) should be replaced by $\left(\varepsilon_{O X}+1\right) / 2$ for the bottom-gate configuration in which the CNTs are exposed to the air. When $d=1.57 \mathrm{~nm}, l=10$, $D=8 \mu \mathrm{m}^{-1}$, and $D^{\prime}=1 \mu \mathrm{m}^{-1}$, the gate capacitance for the dominant CNT in such a bundle was 86 times smaller than that for an individual CNT. Therefore, the gate capacitance was calculated by

$$
C_{O X}=C_{O X i} / \alpha
$$

by introducing a fitting parameter $\alpha$.

\section{E. Drain current}

Figure 8 shows the current paths in a TFT modeled here. It was assumed that the source and drain contacts were ohmic and there were $k$ equivalent current paths each having the number of CNT bundles approximated by $2 \sqrt{2} L / L_{C N T}\left(\equiv n_{C}\right)$. Here, $L$ is the gate length of the TFT and $L_{C N T}$ is the length of CNT bundle. The factor of $2 \sqrt{2}$ comes from the geometrical assumption of orthogonally crossed CNT bundles shown in Fig. 8.

All the CNT bundles and junctions between the bundles were assumed to be equivalent. The voltage drop in each 


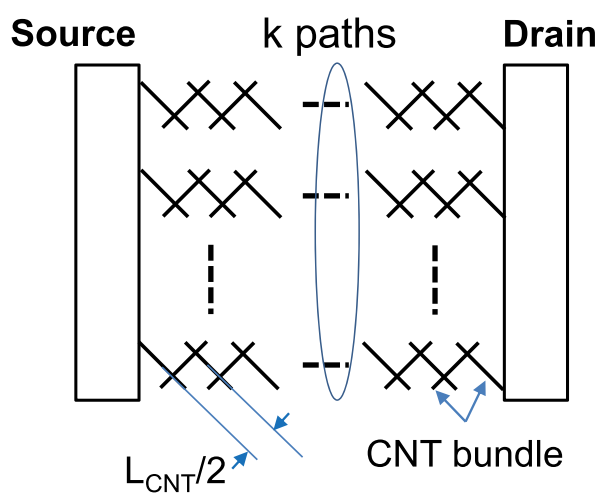

FIG. 8. Current path model for TFT.

CNT bundle $V_{C N T}$ and the Fermi energy difference between the two CNT bundles $\Delta E_{f}$ must satisfy

$$
n_{C} V_{C N T}+\left(n_{C}-1\right) \Delta E_{f}=V_{D S} .
$$

The current through each CNT bundle is expressed by

$$
I_{C N T}=\frac{V_{C N T}}{L_{C N T} / 2} \mu C_{G S}\left(V_{G S}-V_{F B}-\phi_{S}\right),
$$

where $\mu$ is the electron mobility and the surface potential $\phi_{S}$ is determined by the charge neutrality condition (7) in which the Fermi energy $E_{f}$ is assumed to be equal to that in the source $(=0)$. To satisfy the current-continuity condition, the current $I_{C N T}$ must equal the tunnel current through the junction, $I_{T}$, calculated by (5) with $\Delta E_{f}=E_{f L}-E_{f R}$. By selfconsistently solving all the equations described above, the drain current $I_{D S}$ can be obtained as $k I_{C N T}\left(=k I_{T}\right)$.

\section{EVALUATION OF MODEL}

To evaluate the present model, we fabricated bottomgate SWCNT network TFTs on a $\mathrm{SiO}_{2} / \mathrm{Si}$ substrate, where a 200-nm $\mathrm{SiO}_{2}$ and a $\mathrm{Si}$ substrate operated, respectively, as the gate oxide and gate. The TFT channel was formed by repeating 10 cycles of spin-coating SWCNT solution with a semiconducting CNT purity of 99\% (NanoIntegris, Inc., median value of length $=1 \mu \mathrm{m}$ and diameter $=1.2-1.7 \mathrm{~nm}$ ) and washing to remove the surfactant. $\mathrm{Pt} / \mathrm{Pd}$ was used as the source and drain electrodes. The Atomic Force Microscope (AFM) observations revealed that the diameters of CNT bundles ranged from 5.6 to $22.5 \mathrm{~nm}$ and that the bundle density was about $17 \mu \mathrm{m}^{-2}$. The fabricated TFTs exhibited p-channel characteristics. The details of the fabrication method and TFT characteristics will be published elsewhere. ${ }^{23}$

\section{A. Drain current}

In the experiments, the drain currents for the fabricated TFTs gradually changed with time, which might have been caused by hole trapping. When the drain current was measured with sweeping $V_{G S}$, hysteresis phenomena were observed. To avoid the hole trapping effects, the gate voltage was manually changed. A TFT with typical characteristics was used to evaluate the model. The "effective mobility" was extracted by using

$$
g_{m}=\frac{W}{L} C_{G S, p l a t e} \mu_{e f f}\left|V_{D S}\right|
$$

where $C_{G S \text {, plate }}$ is the gate capacitance calculated with a parallel-plate approximation $\left(=\varepsilon_{O X} \varepsilon_{0} / t_{O X}\right)$. In (14), $V_{D S}$ is the applied drain voltage. The extracted $\mu_{\text {eff }}$ was about 1 $\mathrm{cm}^{2} / \mathrm{V} / \mathrm{s}$.

In the calculations, the gate modulation of the potential difference between two CNTs given by (4) was used with taking into account of the difference in the gate oxide thicknesses and gate voltages for the experiments and the threedimensional analysis described in Section II B. The potential distribution shown on the left-hand side of Fig. 5 was used.

Figure 9 compares measured and calculated $I_{D S}-V_{G S}$ characteristics for a TFT with a gate length $L$ of $10 \mu \mathrm{m}$ and width $W$ of $50 \mu \mathrm{m}$. The parameters used in the calculation were as follows: $L_{C N T}=1.0 \mu \mathrm{m}, d=1.49 \mathrm{~nm} \quad(m=19)$, $V_{F B}=-20 \mathrm{~V}, \alpha=155$, and $k=55$. The other parameters were the same as those described above. McEuen et al. reported the SWCNT mobility in the region of 1000-10000 $\mathrm{cm}^{2} / \mathrm{V} / \mathrm{s}^{24}$ In Fig. 9, the solid and dotted lines were, respectively, calculated using $\mu=1000$ and $10000 \mathrm{~cm}^{2} / \mathrm{V} / \mathrm{s}$. The two lines were coincident. When $\mu=1000 \mathrm{~cm}^{2} / \mathrm{V} / \mathrm{s}$, the voltage drop in each CNT bundle $V_{C N T}$ was $17 \mathrm{mV}$. On the other hand, $V_{C N T}$ was $1.7 \mathrm{mV}$ when $\mu=10000 \mathrm{~cm}^{2} / \mathrm{V} / \mathrm{s}$. This suggested that the drain current was mainly determined by the current through the CNT-CNT junction. The used $k$ of 55 means that a current path of $1.1 \mu \mathrm{m}^{-1}$ was formed. The AFM measurements suggested that there were $\sim 10 \mathrm{CNT}$ bundles/ $\mu \mathrm{m}$ when observed in the channel width direction. If one CNT bundle out of 10 contributed to the current conduction, $D^{\prime}$ and $D$ described in Sec. II were 1 and 10, respectively. These gave a value close to 155 for the fitting parameter $\alpha$. A good agreement between measured and calculated characteristics was obtained except in the $V_{G S}$ region close to $V_{F B}$. When $V_{G S}=V_{F B}, \mathrm{n}=\mathrm{p} \sim 2 \times 10^{3}$, which is 4-order smaller than the hole density $p$ at $V_{G S}$ of $-40 \mathrm{~V}$, resulting in 4-order smaller $I_{D S}$. Extending the model to the subthreshold region remains for future work. The experimental "effective mobility" was extracted by using the parallel-plate capacitance and the applied drain voltage. However, the calculations suggested that these treatments

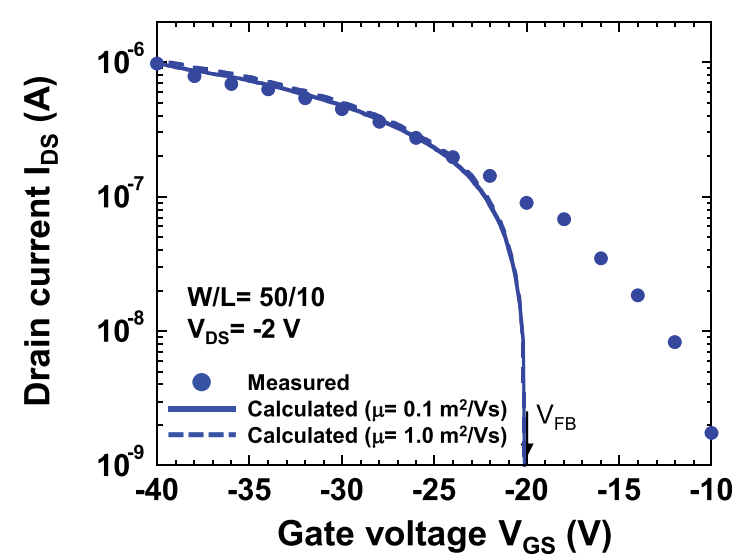

FIG. 9. Comparison between measured and calculated $I_{D S^{-}} V_{G S}$ characteristics for TFT with $L=10 \mu \mathrm{m}$ and $W=50 \mu \mathrm{m}$. 


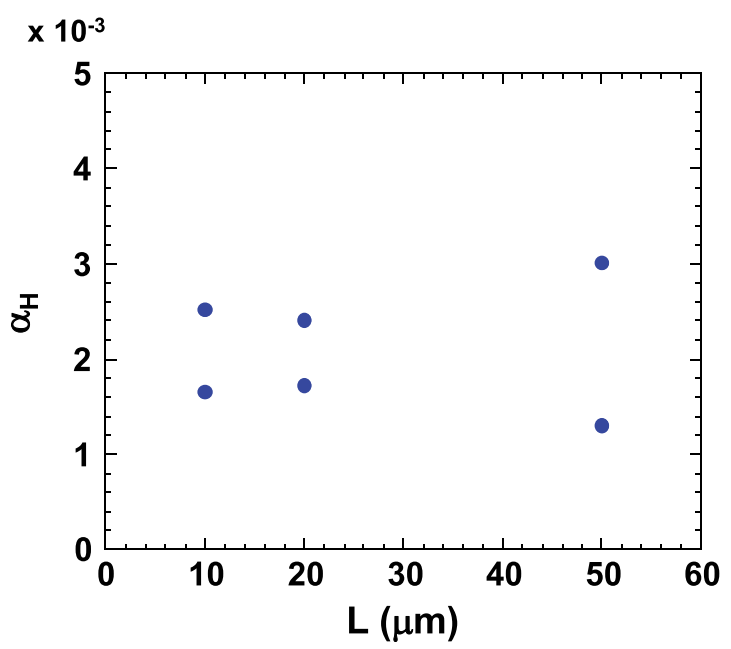

FIG. 10. Derived $\alpha_{H}$.

were incorrect for TFTs. The ratio of the gate oxide capacitance for the TFT $\left(=\sqrt{2} C_{O X i} k L / \alpha\right)$ to the parallel-plate capacitance $\left(=L W C_{G S, \text { plate }}\right)$ was $1 / 385$. The voltage drop in the whole CNT bundles was $1 / 4.2$ times the applied drain voltage in the case of $\mu=1000 \mathrm{~cm}^{2} / \mathrm{V} / \mathrm{s}$. This resulted in the small "effective mobility" in the fabricated TFT $1 / 10^{3}$ times the CNT mobility. The comparison gives information useful for increasing the "effective mobility." To improve the TFT performance, the CNTs with similar bandgaps should be individually and densely dispersed. In addition, the gate length should be reduced and the number of conduction paths should be increased while avoiding the formation of a metallic current path. In these respects, aligned $\mathrm{CNTs}^{22}$ are effective to increase the mobility though the fabrication method is rather complicated.

\section{B. Low frequency noise}

To evaluate the model, we measured the low frequency noise in the drain current of the fabricated TFTs. ${ }^{23}$ The measured noise power density clearly showed $1 / f(f$ : frequency) and $I_{D S}{ }^{2}$ dependencies. Such noise characteristics are well explained by Hooge's empirical relation. In accordance with Hooge, the noise power density is given by ${ }^{25}$

$$
S_{I}=A \frac{I_{D S}^{2}}{f}=\frac{\alpha_{H}}{N} \frac{I_{D S}^{2}}{f},
$$

where $N$ is the number of carriers in the TFT and $\alpha_{H}$ is Hooge's empirical constant $\left(\sim 2 \times 10^{-3}\right)$. The $\alpha_{H}$ was derived from the measured $A$ and calculated $N$. Figure 10 shows the derived $\alpha_{H}$ for six TFTs (W/L $=50 / 10,50 / 20$, and 200/50). These values were close to Hooge's empirical value.

\section{CONCLUSIONS}

We presented a simple model of the drain current in the linear region for CNT network TFTs. The model was based on the gate electrostatics and the continuity condition of the currents through CNT and CNT-CNT tunnel junction. To evaluate the model, we compared the calculated $I_{D S}-V_{G S}$ characteristics to those measured for the fabricated TFT. A good agreement was obtained between measured and calculated characteristics. In addition, the low frequency (1/f) noise parameters $\alpha_{H}$ were derived from the measured noise power densities and the calculated numbers of carriers in TFTs. The derived $\alpha_{H}$ were close to Hooge's empirical value. Even though the present model is based on simplified assumptions, it provides useful information to improve the TFT performance.

\section{ACKNOWLEDGMENTS}

This study was supported by a Grant-in-Aid for Scientific Research from the Ministry of Education, Culture, Sports, Science and Technology, Japan.

${ }^{1}$ R. H. Baughman, A. A. Zakhidov, and W. A. de Heer, Science 297, 787 (2002).

${ }^{2}$ J. Guo, M. Lundstrom, and S. Datta, Appl. Phys. Lett. 80, 3192 (2002).

${ }^{3}$ J. Appenzeller, Proc. IEEE 96, 201 (2008).

${ }^{4}$ E. S. Snow, J. P. Novak, P. M. Campbell, and D. Park, Appl. Phys. Lett. 82, 2145 (2003)

${ }^{5}$ S.-H. Hur, C. Kocabas, A. Gaur, O. O. Park, M. Shim, and J. A. Rogers, J. Appl. Phys. 98, 114302 (2005).

${ }^{6}$ S. Kumar, J. Y. Murthy, and M. A. Alam, Phys. Rev. Lett. 95, 066802 (2005).

${ }^{7}$ P. Beecher, P. Servati, A. Rozhin, A. Colli, V. Scardaci, S. Pisana, T. Hasan, A. J. Flewitt, J. Robertson, and G. W. Hsieh, J. Appl. Phys. 102, 043710 (2007).

${ }^{8}$ C. Kocabas, N. Pimparkar, O. Yesilyurt, S. J. Kang, M. A. Alam, and J. A. Rogers, Nano Lett. 7, 1195 (2007).

${ }^{9}$ M. A. Topinka, M. W. Rowell, D. Goldhaber-Gordon, M. D. McGehee, D. S. Hecht, and G. Gruner, Nano Lett. 9, 1866 (2009).

${ }^{10}$ C. Wang, J. Zhang, K. Ryu, A. Badmaev, L. G. de Arco, and C. Zhou, Nano Lett. 9, 4285 (2009).

${ }^{11}$ D.-M. Sun, M. Y. Timmermans, Y. Tian, A. G. Nasibulin, E. I. Kauppinen, S. Kishimoto, T. Mizutani, and Y. Ohno, Nat. Nanotechnol. 6, 156 (2011).

${ }^{12}$ C. Wang, A. Badmaev, A. Jooyaie, M. Bao, K. L. Wang, K. Galatsis, and C. Zhou, ACS Nano 5, 4169 (2011).

${ }^{13}$ M. S. Fuhrer, J. Nygard, L. Shih, M. Forero, Y.-G. Yoon, M. S. C. Mazzoni, H. J. Choi, J. Ihm, S. G. Louie, A. Zettl, and P. L. McEuen, Science 288, 494 (2000).

${ }^{14}$ S. Datta, Quantum Transport: Atom to Transistor (Cambridge University Press, Cambridge, 2005).

${ }^{15}$ S. Suzuki, C. Bower, Y. Watanabe, and O. Zhou, Appl. Phys. Lett. 76, 4007 (2000).

${ }^{16}$ T. Tanaka, K. Mori, E. Sano, B. Fugetsu, and H. Yu, Physica E 44, 997 (2012).

${ }^{17}$ P. N. Nirmalraj, P. E. Lyons, S. De, J. N. Coleman, and J. J. Boland, Nano Lett. 9, 3890 (2009).

${ }^{18}$ A. Znidarsic, A. Kaskela, P. Laiho, M. Gaberscek, Y. Ohno, A. G. Nasibulin, E. I. Kauppinen, and A. Hassanien, J. Phys. Chem. C 117, 13324 (2013).

${ }^{19}$ E. M. Doherty, S. De, P. E. Lyons, A. Shmeliov, P. N. Nirmalraj, V. Scardaci, J. Joimel, W. J. Blau, J. J. Boland, and J. N. Coleman, Carbon 47, 2466 (2009)

${ }^{20}$ V. Scardaci, R. Coull, and J. N. Coleman, Appl. Phys. Lett. 97, 023114 (2010).

${ }^{21}$ M. Jaiswal, W. Wang, K. A. Shiral Fernando, Y.-P. Sun, and R. Menon, J. Phys.: Condens. Matter 19, 446006 (2007).

${ }^{22}$ S. J. Kang, C. Kocabas, T. Ozel, M. Shim, N. Pimparkar, M. A. Alam, S. V. Rotkin, and J. A. Rogers, Nat. Nanotechnol. 2, 230 (2007).

${ }^{23}$ T. Tanaka and E. Sano, "Low-frequency noise in carbon nanotube network thin-film transistors" (unpublished).

${ }^{24}$ P. L. McEuen, M. S. Fuhrer, and H. Park, IEEE Trans. Nanotechnol. 1, 78 (2002).

${ }^{25}$ F. N. Hooge, Phys. Lett. A 29, 139 (1969). 